\title{
Modular Localization and the Bootstrap-Formfactor Program
}

\author{
Bert Schroer \\ Institut für Theoretische Physik, FU-Berlin, Arnimallee 14, 14195 Berlin \\ e-mail : schroer@physik.fu-berlin.de \\ Temporary adress: CBPF, Rio de Janeiro, Brazil \\ e-mail:schroer@cat.cbpf.br \\ June 15, 2018
}

\begin{abstract}
We extend the "modular localization" principle from free to interacting theories and test its power for the special class of $d=1+1$ factorizing models.
\end{abstract}

\section{Contents}

\begin{tabular}{lll}
\hline 1 & Introduction & 2
\end{tabular}

\begin{tabular}{|lll}
2 & Modular Localization and Interaction & 6
\end{tabular}

3 The Bootstrap-Formfactor Program 8

3.1 Properties of Factorizing S-Matrices . . . . . . . . . . . . . . . 9

3.2 Generalized Formfactors . . . . . . . . . . . . . . . . . . . . . 13

3.3 Modular Theory and the Formfactor Program . . . . . . . . . 17

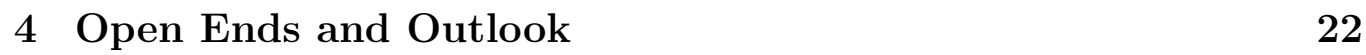




\section{Introduction}

Presently QFT presents itself as being formed of several parts which seem to drift apart into different directions. On the one hand there is the standard approach which is centered around renormalized perturbation theory and the various quantization methods (canonical, functional). Enriched with geometrical ideas it has led to recent (mainly) mathematical advances via string theory and the Seiberg-Witten duality structure. On the other hand there is the more algebra-based low dimensional approach which has led to the construction of rich families of chiral conformal and integrable QFT. The latter approach, although being somewhat conservative in its use of physical principles, has nevertheless led to many startling results concerning e.g. fusion of antiparticles from particles, confined objects and solitons as being two sides of the same coin, and other extended (and somewhat surprising) manifestations of the principle of "nuclear democracy". Thirdly there is a very small group of theoreticians who find it profitable to continue the structural investigations of algebraic QFT.

In fact the most interesting message of the low dimensional constructive bootstrap-formfactor program seems to be that the emphasis on the scattering matrix advocated way back by Heisenberg and later by Chew, Stapp and others, was well founded. What went wrong in those early attempts was related to the enforced and artificial separation from local QFT and the (cyclically recurrent) ideologically motivated working hypothesis of a theory of everything (in this case: everything minus gravity). The main theme of this article is the realization that the S-matrix in algebraic QFT acquires a new hitherto unknown pivotal role in the construction of local nets (whose generators are local fields). It belongs to the foundation of the local field theory (in its role as the net invariant which carries local modular information) as well as to its roof (in its role as describing scattering observables), a truly vexing "bootstrap" situation. The fact that in $d=1+1$ factorizable theories Chew's bootstrap ideas for the S-matrix work without fields (but with the help of "fusion" and "Yang-Baxter") is not due to the correctness of the underlying philosophy but rather to undeserved luck: the physical rapidity scattering variable is at the same time the uniformization parameter of the analytic properties 1 . In higher dimensions or without the factorization,

\footnotetext{
${ }^{1}$ Even in $\mathrm{d}=1+1$ the situation is very far removed from the desired uniqueness of Chew's S-matrix approach.
} 
Chew's program would fail without the use of local fields (and it did fail). In that case an iterative procedure which corrects the S-matrix together with a locality improvement of states and fields may have a constructive chance, a situation which could be vaguely reminiscent of the Hartree-Fock iteration in Schrödinger theory..

In this note we propose a new concept [1] of "modular localization" which, as will be shown, is capable of reconquering the lost unity of QFT. In particular, we will learn a new and very interesting lesson from the $\mathrm{d}=1+1$ formfactor program. Far from being a special "exotic" construction, remote from any "real" QFT, this approach, if analyzed with general and deep concepts related to the TCP theorem and the S-matrix (interpreted as an invariant of a local net), reveals a surprising new and powerful nonperturbative construction principle which, so we hope, may turn out to be the basis of a future new iterative constructive approach. Locality of observables and localization of states (always relative to the vacuum or some other distinguished reference state) in QFT is a conditio sine qua non for the physical interpretation (without any outside impositions). Global topology as in the so called "topological field (?) theories" or the vacuum structure assigned to certain effective potentials which does not result from the local structure of real time QFT remain part of mathematics.

The fastest way to get a glimpse at the "modular localization" is to look at the relation of the Wigner representation theory [2] for positive energy representations of the Poincaré group and free fields. Whereas in Wigner's theory these irreducible representations in $H_{W i g}^{(m, s)}$ are uniquely specified by their mass and their spin (resp. helicity), this uniqueness is lost if one passes to free fields in the Fock-space $H_{F}^{(m, s)}$. There are infinitely many free fields in Fock-space and they constitute the linear part (in creation and annihilation operators) of a huge local equivalence class of fields, the so called Borchers class $B(m, s)$ 3] Any cyclic (with respect to the vacuum) representative field from this class generates the same net of local von Neumann algebras in $H_{F}$ :

$$
\mathcal{O} \rightarrow \mathcal{A}(\mathcal{O})
$$

In fact the emerging picture of pointlike fields, that behave similar to coordinates in differential geometry, was the prime motivation for formulating algebraic QFT in terms of nets of algebras [3]. In our illustrative example [1] we regained the lost Wigner unicity on the level of nets. For a detailed presentation of the physical motivations and aims of this algebraic QFT we refer 
to a forthcoming article in Annals of Physics [⿴囗十

For the following it is important to understand the direct construction of this net in terms of the "modular localization" principle. For that one uses the operators which Wigner's theory affiliates with a reference wedge for which we take the $\mathrm{x}$-t wedge:

$$
\begin{aligned}
W_{r} & :|t|<x \\
\delta^{i t} & \equiv U\left(\Lambda_{r}(2 \pi t)\right), \quad j \equiv U\left(r_{r}\right)
\end{aligned}
$$

Here $\Lambda_{r}(\chi)$ and $r_{r}$ are the $\mathrm{x}$-t Lorentz-boost and the x-t reflection. The latter is represented by a antiunitary operator $j$ related (via a $\pi$-rotation around the $\mathrm{x}$-axis) to the TCP transformation $\vartheta$. For a charged particle this requires doubling of the Wigner space $H_{W i g}^{(m, s)}$. The reflection commutes with the L-boost. It has been shown elsewhere [5] [1] that the unbounded antilinear involution:

$$
s=j \delta^{\frac{1}{2}}
$$

from which $j$ and $\delta^{\frac{1}{2}}$ can be recovered by polar decomposition, can be used in order to define a real subspace $H_{R}$ :

$$
H_{R}=\left\{\psi \in H_{W i g} \mid s \psi=\psi\right\}
$$

The momentum space wave functions in $H_{R}$ are in the domain of $\delta^{\frac{1}{2}}$ and hence have analytic properties in the rapidity variable $\theta$ associated to the standard wedge $\mathrm{W}: p_{r}=\left(|p| \cosh \theta,|p| \sinh \theta, p_{2}, p_{3}\right),|p|=\left(m^{2}+p_{2}^{2}+p_{3}^{2}\right)^{\frac{1}{2}}$. For selfconjugate particles $H_{R}$ consists of analytic functions in the strip $0<\theta$ $<\pi$ for which the two boundary values are related by a generalized reality condition:

$$
D^{(s)}\left(i \sigma_{2}\right) \underset{\chi \rightarrow i \pi}{a . c .}\left[D^{(s)}\left(R_{W}\left(\Lambda_{r}(\chi), p_{r}\right)\right] \psi\left(\theta+i \pi, p_{2}, p_{3}\right)=\overline{\psi\left(\theta, p_{2}, p_{3}\right)}\right.
$$

where $\psi$ is the $2 \mathrm{~s}+1$ component Wigner wave function, $R_{W}\left(\Lambda_{r}, p\right)$ the Wigner rotation associated with the boost $\Lambda$ on which the analytic continuation a.c. acts, and $D^{(s)}\left(i \sigma_{2}\right)$ the charge conjugation matrix. In the non-selfconjugate case (particles $\neq$ antiparticles) the reflection $j$ involves a flip in a doubled Wigner space. $H_{R}$ has a property which is called "standard" i.e.:

$$
H_{R} \cap i H_{R}=\{0\}, \quad H_{R}+i H_{R} \text { dense in } H_{W i g}
$$


and $\mathrm{j}$ transforms $H_{R}$ into its symplectic complement $H_{R}^{\prime}$ (the symplectic form is the imaginary part of the scalar product in $H_{W i g}$ ) which in the case of integer spin representations (the modifications for halfinteger spin are explained in [1]) is the same as the geometric opposite wedge space:

$$
\begin{aligned}
& H_{R}^{\prime} \equiv H_{R}\left(W_{r}\right)^{\prime}=H_{R}\left(W_{r}^{\prime}\right) \\
& W_{r}^{\prime} \equiv W_{r}^{\text {opposite }}, \quad H_{R}(W) \cap H_{R}\left(W^{\prime}\right)=\{0\}
\end{aligned}
$$

$\mathcal{P}$-covariance generates from $W_{r}$ a family of wedges $W=g W_{r}$ and associated $s(W), j(W), \delta(W)$ and $H_{R}(W)$ with the net isotony property:

$$
H_{R}\left(W_{1}\right) \subset H_{R}\left(W_{2}\right) \quad W_{1} \subset W_{2}
$$

where the properness of the inclusion is a consequence of the positivity of the energy (in fact equivalent to it, as it turns out) [5]. Wedge localization in the Wigner theory (not to be confused with the Newton-Wigner localization) [1] is the statement that the dense subspace (the precise domain of $s$ ) $H_{R}+i H_{R}$ describes localization inside the reference wedge. From QFT it is known that if one applies smeared local fields $\phi$ with test functions which are supported in $W_{r}$ to the vacuum, one obtains a dense set of $W$-localized state vectors. The relation between the field algebras restricted to wedges and the TomitaTakesaki modular theory was first observed by Bisognano and Wichmann [6] and later used by Sewell [7] in order to obtain a structural understanding of the Hawking-Unruh effect. What is new is that these spaces allow (in the present case for free systems) for a very neat characterization in terms of closed real subspaces whose position within the total space contains the full information about localization regions. Localization inside compact regions viz. double cones $\mathrm{C}$ (which are inaccessible by direct geometric modular theory) may be defined in terms of (dense if nontrivial) intersections:

$$
H_{R}(C)=\bigcap_{W \supset C} H_{R}(W)
$$

As already mentioned, the double cone localization is fulfilled in all positive energy representations with halfinteger spin, but not in $\mathrm{d}=3+1 \mathrm{~m}=0$ "continuous spin" [1] representation and also not in massive $\mathrm{d}<3+1$ representations with "anyonic"spin.

The important last step in the construction of a localized net of von Neumann algebras is the application of the CCR (Weyl) and the CAR functor 
which maps the net of real Hillbert subspaces into the net of algebras [5] [1]

$$
\begin{aligned}
H_{W i g} & \rightarrow B\left(H_{F}\right)=\operatorname{alg}\left\{W \operatorname{eyl}(f) \mid f \in \mathcal{S}\left(M_{4}\right)\right\} \\
H_{R}(W) & \rightarrow \mathcal{A}(W)=\operatorname{alg}\{W \operatorname{eyl}(f) \mid f \in \mathcal{D}(W)\} \\
\check{S} & =J \Delta^{\frac{1}{2}}, \quad J=e^{j}, \quad \Delta=e^{\delta}
\end{aligned}
$$

These operators $\check{S}, J$ and $\Delta^{i t}$ are the Tomita-Takesaki operators of the T-T modular theory of the von Neumann algebra $\mathcal{A}(W)$ in "standard position". The inverse hat $\vee$ on $\mathrm{S}$ helps to distinguish the Tomita involution from the later appearing scattering S-operator, usually referred to as the S-matrix.

For the somewhat subtle point that the obstruction against the equality of the geometric opposite with the modular opposite localization in the case of halfinteger spin requires the introduction of a "Klein twist" and the CAR functor we refer to the mentioned literature [1].

\section{Modular Localization and Interaction}

In order to obtain a clue of how to incorporate an intrinsic notion of interactions into this modular localization setting, we remind ourselves that if we do use pointlike fields, the modular localization for free fields agrees with what we get by applying the polynomial in the localization region supported smeared fields. In contrast to the conventional characterization of localization in terms of x-space pointlike fields, the modular characterization solely works in the momentum-(Fock)space of the (incoming) free particles. It attributes a physical significance to the precise position of the Reeh-Schlieder [3] dense set of localized vectors and the change of this position resulting from the change of localization region. In order to formulate the modular localization principle in the case of interactions, one must take note of the fact that the scattering matrix $\mathrm{S}$ of local QFT is the product of the interacting TCP $\Theta$ with the free (incoming) TCP $\Theta_{0}$ and (since the rotation by which the Tomita reflection $J$ differs from $\Theta$ is interaction-independent as all connected Poicaré transformations are interaction-independent) we have:

$$
S=\Theta \cdot \Theta_{0}, \quad S=J \cdot J_{0}
$$

and as a result we obtain for the Tomita involution:

$$
\check{S}=J \Delta^{\frac{1}{2}}=S J_{0} \Delta^{\frac{1}{2}}=S \check{S}_{0}
$$


Again we may use covariance in order to obtain $\check{S}(W)$ and the localization domain of $\check{S}(W)$ as $\mathcal{D}(\check{S}(W))=H_{R}(W)+i H_{R}(W)$ i.e. in terms of a net of closed real subspaces $H_{R}(W)$ of the incoming Fock space. However now the construction of an associated von Neumann algebra is not clear since an "interacting" functor from subspaces of the Fock space to von Neumann algebras is not known. We will make some remarks (still short of a solution of this important problem) in the concluding section and continue here with some more helpful comments on modular localization of interacting state vectors.

As in the free case, the modular wedge localization does not use full Einstein causality but only the so-called "weak locality", which is just a reformulation of the TCP invariance [8] Weakly local fields form an equivalence class which is much bigger than the local Borchers class but they are still associated to the same S-matrix (or rather the same TCP operator). Actually the $\mathrm{S}$ in local quantum physics has two different interpretations: $\mathrm{S}$ in its role to provide modular localization in interacting theories, and $\mathrm{S}$ with the standard scattering interpretation in terms of (nonlocal!) large time limits. There is no parallel outside local quantum physics to this state of affairs. Whereas all concepts and properties which have been used hitherto in standard QFT (perturbation theory, canonical formalism and path integrals) as e.g. time ordering [ and interaction picture formalism, are shared by nonrelativistic theories, modular localization is a new structural element in local quantum physics of and a characteristic property for Einstein causal quantum physics. The simplest kind of interacting theories are those in which the particle number is at least asymptotically ("on shell") conserved i.e. $\left[\check{S}, \mathbf{N}_{i n}\right]=0$.

In the next section we will briefly review the $\mathrm{d}=1+1$ bootstrap-formfactor program in a manner which facilitates the later application of modular localization.

\footnotetext{
${ }^{2}$ There is a conspicuous absence of the time-ordering operation in the bootstrap construction of factorizable field models. Instead the basic objects are generalized formfactors i.e. sesquilinear forms on a dense set of state vectors.

${ }^{3}$ This characteristic modular structure lifts local quantum physics to a new realm by its own which cannot be obtained by specialization from general quantum theory.
} 


\section{The Bootstrap-Formfactor Program}

In this section we will meet a constructive approach for "integrable" $d=1+1$ QFT. Our first task is to obtain an intrinsic QFT understanding of integrability in a way which avoids classical notions as e.g. complete sets of conservation laws etc. For this purpose we note an important $\mathrm{d}=1+1$ peculiarity.. Our generic expectation is that large spatial separation of the center of wave packet of two particles in the elastic two-particle scattering matrix leads to the weakening of scattering, or in momentum space:

$$
\left\langle p_{1}^{\prime} p_{2}^{\prime}|S| p_{1} p_{2}\right\rangle=\left\langle p_{1}^{\prime} p_{2}^{\prime} \mid p_{1} p_{2}\right\rangle+\delta\left(p_{1}+p_{2}-p_{1}^{\prime}-p_{2}^{\prime}\right) T\left(p_{1} p_{2} p_{1}^{\prime} p_{2}^{\prime}\right)
$$

where the identity contribution is more singular (has more $\delta$-factors) than the T-term and therefore the second term drops out in x-space clustering. This argument fails precisely in $\mathrm{d}=1+1$ and therefore the cluster property of the S-matrix is not suitable in order to obtain an intrinsic understanding of interaction. The two-particle S-matrix looses its higher particle threshold structure, but it remains nontrivial (in distinction to $\mathrm{d}=3+1$ ). However for all higher particle scattering processes the behavior for $d=1+1$ is qualitatively the same as in higher dimensions: the decreasing threshold singularities (which decrease with increasing particle number) are responsible for the spatial decrease. Therefore any $\mathrm{d}=1+1$ QFT is expected to have a limiting $\mathrm{S}_{\text {lim }}$-matrix which is purely elastic and solely determined by the elastic two-particle $S^{(2)}$-matrix. The Yang-Baxter relation results as a consistency relation for the elastic $3 \rightarrow 3$ particle $S_{\text {lim}}^{(3)}$-matrix. If this limiting S-matrix would again correspond to a localizable QFT, we would have a new class division of QFT, this time based on a long distance limit (which in some sense is opposite to the scale invariant short distance limit). It is this (long distance) class property ${ }^{4}$ which makes these factorizing models so fascinating, as much as the fascination of chiral conformal QFT results from their role of representing short distance universality classes. In $\mathrm{d}=3+1 \mathrm{~S}_{\mathrm{lim}}=1$ and therefore the limiting theory is expected to maintain the same superselection rules but in the "interaction freeest" possible way (literally free theories as we will argue later on). Hence in $d=1+1$ we are invited to speculate on the

\footnotetext{
${ }^{4}$ Although I know of no article in which this has been spelled out, its pervasive presence behind the scene is is recognizable in some publications.
} 
validity of the following commutative diagram:

$$
\mathcal{F} \searrow \begin{aligned}
& \mathcal{F}_{l d} \\
& \stackrel{\mathcal{F}}{\mathcal{F}}_{s d}
\end{aligned}
$$

Here $l d(s d)$ labels the long (short) distance limits. There are also arguments [9] that with the help of a perturbative idea one may ascend from $\mathcal{F}_{s d}$ to $\mathcal{F}_{l d}$. It is however presenly not clear how one can use the known properties of the $l d$ theories (i.e. integrable models) in order to formulate a constructive program for the nonintegrable members of the $l d$ equivalence class. We hope that our modular localization principle (which is not restricted to factorizable models) may turn out to be helpful for this purpose.

The constructive approach based on the bootstrap idea proceeds in two steps. One first classifies unitary, crossing symmetric solutions of the YangBaxter equations which fulfill certain minimal (or maximal, depending on the viewpoint) requirements. Afterwards we use these factorizing S-matrices together with the Watson equations (a notion from scattering theory relating formfactors with the S-matrix) and analytic properties for formfactors in order to compute the latter. One obtains the complete set of multi-particle matrix elements of "would be" local fields, i.e. one constructs the fields as sesquilinear forms. It is characteristic of this method that one does not use the "axiomatic" properties of the beginning of this section but rather less rigorously known momentum-space analytic properties which, although certainly related to causality and spectral properties, are more part of the $\mathrm{LSZ}+$ dispersion theoretic folklore than of rigorous QFT. As long as one demonstrates at the end that the so obtained fields fulfill local commutativity this is a legitimate procedure. It leaves open the question whether there exists a more direct conceptual link between the S-matrix and the local fields or rather the field independent local nets. That this is indeed the case will be shown after the presentation of the formfactor program.

\subsection{Properties of Factorizing S-Matrices}

Consider first the analytic structure of an elastic S-matrix for a scalar neutral particle. In terms of the rapidity variable $\theta$ :

$$
\left|p_{1,} p_{2}\right\rangle^{\text {out }}=S\left|p_{1}, p_{2}\right\rangle^{\text {in }}=S_{e l}\left(p_{1}, p_{2}\right)\left|p_{1}, p_{2}\right\rangle^{\text {in }}
$$




$$
\begin{aligned}
S_{e l}\left(p_{1}, p_{2}\right) & =: S(\theta), \quad p_{i}=m\left(\cosh \theta_{i}, \sinh \theta_{i}\right), \quad \theta:=\mid \theta_{1}-\theta(1 \mid 5) \\
{ }^{i n}\left\langle p_{1}^{\prime}, p_{2}^{\prime}|S| p_{1}, p_{2}\right\rangle^{i n} & =S(\theta)^{i n}\left\langle p_{1}^{\prime}, p_{2}^{\prime} \mid p_{1}, p_{2}\right\rangle^{i n}
\end{aligned}
$$

Usually the elastic S-matrix is written in terms of the invariant energy $s=$ $\left(p_{1}+p_{2}\right)^{2}=2 m^{2}(1+\cosh \theta)$ and the momentum transfer (not independent in $\mathrm{d}=1+1) t=\left(p_{1}-p_{2}\right)^{2}=.2 m^{2}(1-\cosh \theta)$. As a result of undeserved fortune, the rapidity $\theta$ turns out to be a uniformization variable for the real analytic $\mathrm{S}$ i.e. the complex s-plane with the elastic cut in $s \geq 4 \mathrm{~m}^{2}$ is dumped into the strip $0 \leq \operatorname{Im} \theta \leq \pi$ and the S-matrix becomes a meromorphic function $S(\theta)$ with $S(-\theta)=S^{*}(\theta)=S^{-1}(\theta)$. (unitarity). Hence the strip $-\pi \leq \theta \leq \pi$ is the physical strip for $S(\theta)$. Crossing symmetry in our special (neutral) case means a symmetry on the boundary of the strip: $\theta \rightarrow i \pi-\theta$. Note that the presence of inelastic thresholds would destroy the uniformization.

The factorization implies the operator relation:

$$
\begin{aligned}
& S_{12}\left(p_{1}, p_{2}\right) S_{13}\left(p_{1}, p_{3}\right) S_{23}\left(p_{2}, p_{3}\right) \\
= & S_{23}\left(p_{2}, p_{3}\right) S_{13}\left(p_{1}, p_{3}\right) S_{12}\left(p_{1}, p_{2}\right)
\end{aligned}
$$

According to Liouville's theorem, the only minimal solution (minimal number of poles,smallest increase at $\infty$ ) for this scalar diagonal case is $S= \pm 1$. More general solutions are obtained by placing bound-state poles into the minimal solution. In order to maintain unitarity, the pole factor must be of the form:

$$
P(\theta)=\frac{\sinh \theta+i \sin \lambda}{\sinh \theta-i \sin \lambda}
$$

Transforming back this pole at $\theta=i \lambda$ into the original individual particle variables, we obtain the following parametrization in terms of a center of mass and relative rapidity:

$$
\begin{aligned}
& p_{1}=m\left(\cosh \left(\chi+\frac{i \lambda}{2}\right), \sinh \left(\chi+\frac{i \lambda}{2}\right)\right) \\
& p_{2}=m\left(\cosh \left(\chi-\frac{i \lambda}{2}\right), \sinh \left(\chi-\frac{i \lambda}{2}\right)\right)
\end{aligned}
$$

Clearly the two-particle bound state has the momentum:

$$
\begin{aligned}
& p_{1,2}=\left(p_{1}+p_{2}\right)_{\text {at bd.state }}=2 m \cos \frac{\lambda}{2}(\cosh \chi, \sinh \chi) \\
& p_{1,2}^{2}=m_{2}^{2}, \quad m_{2}=\frac{m}{2 \sin \frac{\lambda}{2}} \sin \lambda
\end{aligned}
$$


The "fusion" of particles may be extended. For a 3-particle bound state we would look at the 3-particle S-matrix which, as a result of factorization has the form:

$$
S^{(3)}\left(p_{1}, p_{2}, p_{3}\right)=S\left(\theta_{12}\right) S\left(\theta_{13}\right) S\left(\theta_{23}\right)
$$

We first fuse 1 with 2 and simultaneously 2 with 3 as before. The center of mass + relative rapidity parametrization yields:

$$
\begin{aligned}
& p_{1}=m(\cosh (\chi+i \lambda), \sinh (\chi+i \lambda)) \\
& p_{2}=m(\cosh \chi, \sinh \chi) \\
& p_{3}=m(\cosh (\chi-i \lambda), \sinh (\chi-i \lambda))
\end{aligned}
$$

Again we get the mass of the 3-particle bound state by adding the zero components in the $\chi=0$ frame:

$$
m_{3}=\left(p_{1}+p_{2}+p_{3}\right)_{0}=m_{2} \cos \frac{\lambda}{2}+m \cos \lambda=2 \frac{m}{2 \sin \frac{\lambda}{2}} \sin \frac{3 \lambda}{2}
$$

Induction then gives the general fusion mass formula:

$$
m_{n}=2 \mu \sin \frac{n \lambda}{2}, \quad \mu=\frac{m}{2 \sin \frac{\lambda}{2}}
$$

We will meet such trigonometric fusion formulas later in algebraic QFT where they are related to the statistical dimensions of fused charge sectors. They were first known through the Dashen-Hasslacher-Neveu quasiclassical approach. The above fusion calculation was done as far back as 1976 [10] and consisted in a synthesis of the quasiclassical work of DHN with some ideas of Sushko using the factorization principle, but still without the ideas of Yang and Baxter (which are not needed for this scalar case). The decisive step towards a general factorizable bootstrap program was taken two years later |11] [12.

The consistency of these particles as incoming and outgoing objects leads to additional structures. Consider the scattering of the mass $m_{2}$ bound state with a third m-particle. This S-matrix for the scattering of these two different particles is obtained from $S^{(3)}$ by:

$$
\left.S_{b . e .}\left(p_{1}+p_{2}, p_{3}\right)\right|_{\left(p_{1}+p_{2}\right)^{2}=m_{2}^{2}}=\frac{1}{R} \underset{\left(p_{1}+p_{2}\right)^{2} \rightarrow m_{2}^{2}}{R e s} S_{12} S_{13} S_{23}
$$


where the projector $P_{12}$ together with a numerical residue value $R$ is defined by:

$$
\underset{\left(p_{1}+p_{2}\right)^{2} \rightarrow m_{2}^{2}}{\operatorname{Res}} S\left(p_{1}, p_{2}\right)=R P_{12}
$$

and we used the word elementary e.and bound $b$. as labels on the new twoparticle $S_{\text {b.c. }}$. The factorization insures that:

$$
P_{12} S_{13} S_{23}=S_{23} S_{13} P_{12}
$$

A prominent family of scalar S-matrices with $\mathrm{N}-1$ bound state fulfilling all these requirements are the $Z_{N}$ models [13]. Consistency requires that the bound state of $\mathrm{N}-1 \mathrm{~m}$-particles is again a m-particle. For $\mathrm{N}=2$ this family contains the Ising field theory with $S_{\text {Ising }}^{(2)}=-1$ which we already met in the section on (dis)order variables. Instead of elaborating this scalar factorization situation, we pass immediatly to the matrix case where we meet a new and interesting phenomenon. We assume that the particle from which we start has an internal "charge" which can take on a finite number of values i.e.

$$
|p, \alpha\rangle \in H_{1} \otimes V, \quad \operatorname{dim} V<\infty
$$

The two-particle S-matrix is then written as a matrix acting on $V \otimes V$ whose entries are operator-valued (represented as in the previous case by momentum-space kernels):

$$
\begin{gathered}
S\left|p_{1}, \ldots, p_{n}\right\rangle_{\alpha_{1} \ldots \alpha_{n}}^{i n}=\left|p_{1}, \ldots, p_{n}\right\rangle_{\alpha_{1}^{\prime} \ldots \alpha_{n}^{\prime}}^{i n} S_{\alpha_{1} \ldots \alpha_{n}}^{\alpha_{1}^{\prime} \ldots \alpha_{n}^{\prime}}\left(p_{1}, \ldots . p_{n}\right) \\
S^{(n)}\left(p_{1}, \ldots, p_{n}\right)=\prod_{i<j} S^{(2)}\left(p_{i}, p_{j}\right)
\end{gathered}
$$

The factorization requires a specific order of the product of matrices. Consistency requires the validity of a Artin (braid-group) like relation:

$$
\begin{aligned}
& S_{12}\left(p_{1}, p_{2}\right) S_{13}\left(p_{1}, p_{3}\right) S_{23}\left(p_{2}, p_{3}\right) \\
= & S_{23}\left(p_{2}, p_{3}\right) S_{13}\left(p_{1}, p_{3}\right) S_{12}\left(p_{1}, p_{2}\right)
\end{aligned}
$$

The notation should be obvious: the subscript on $\mathrm{S}$ indicates on which of the tensor factors in the 3-fold tensor product of one-particle spaces the object acts. The relation with the Artin relations becomes clear if one ignores the p-dependence and rewrites the Y-B relation in terms of $\tilde{S} .=P S$, where $\mathrm{P}$ is the permutation of two tensor factors. 
This is the famous Yang-Baxter relation, since at the time of the discovery of the S-matrix bootstrap it became clear that such a mathematical structure had appeared before outside QFT in a quite different setting. Here this identity permits to change the temporal order of individual rescatterings so that the n-particle scattering $S^{(n)}$ is independent of those (graphically: invariance under parallel shifts of 2-momenta in graphical illustrations of scattering processes). The problem of finding the natural parametrization (e.g. Baxter's elliptic parametrization) for these Yang-Baxter relations does not arise in QFT; the uniformizing rapidity $\theta$ is already the natural YangBaxter variable:

$$
S_{12}(\theta) S_{13}\left(\theta+\theta^{\prime}\right) S_{23}\left(\theta^{\prime}\right)=S_{23}\left(\theta^{\prime}\right) S_{13}\left(\theta+\theta^{\prime}\right) S_{12}(\theta)
$$

If fermion-antifermion pairs can go into boson-antiboson pairs, the object which fulfills the Yang-Baxter relation is not $\mathrm{S}$ but $\sigma S$ where $\sigma= \pm 1$ with + for bosons. As the braid group relation, this is an overdetermined system of equations. For the former one found a powerful mathematical framework within V.Jones subfactor theory [14]. Although the attempts to get an equally powerful mathematical framework for the latter was less than successful (the "Baxterization" of the subfactor representations of Artin braids) one was able to find many interesting families of nontrivial solutions of which some even allowed a comparision with Lagrangian perturbation theory.

The S-matrix bootstrap idea originated in the early $60^{i e s}$ from dispersion theory. Its revival in connection with $d=1+1$ factorization in the late $70^{\text {ies }}$ showed that its premises were physically reasonable, except the idea that it could be seen as a "theory of everything" (TOE) which was wrong and even obsert (for the more recent TOE's one would be hard pressed to say friendly words about their physical content).

The basic new message [15] 16] is that one should use these factorizing Smatrices as computational tools for the construction of local fields and local nets as explained in the following subsection

\subsection{Generalized Formfactors}

Now we will probe the idea that these S-matrices belong to localizable fields. Let A be any local field which belongs to a Borchers equivalence class of local fields. We write the generalized formfactor of $\mathrm{A}(\mathrm{x})$ as:

$$
{ }_{\alpha_{1} \ldots \alpha_{m}}^{\text {out }}\left\langle p_{1}, \ldots, p_{m}|A(0)| p_{m+1}, \ldots, p_{n}\right\rangle_{\alpha_{m+1} \ldots \alpha_{n}}^{i n}
$$


We are interested in its analytic p-space properties. "On shell" p-space analytic properties are more elusive than x-space analytic properties. For the latter the spectral support properties play the important role, whereas p-space analyticity relies heavily on caucality. The above matrix element still contains energy-momentum $\delta$-functions resulting from contracting incoming p's with outgoing. These are removed by taking the connected parts of the formfactors. Only for the distinguished formfactor:

$$
\left\langle 0|A(0)| p_{1}, \ldots p_{n}\right\rangle_{\alpha_{1} \ldots \alpha_{n}}^{i n}=\left\langle 0|A(0)| p_{1}, \ldots . p_{n}\right\rangle_{\alpha_{1} \ldots . \alpha_{n}}^{i n, c o n}
$$

we have coalescence with its connected part. Similar to x-space analyticity, one expects the existence of one analytic master-function whose different boundary values correspond to the different n-particle formfactors:

$$
\begin{aligned}
& \alpha_{1} \ldots \alpha_{m}{ }^{\text {out }}\left\langle p_{1}, \ldots, p_{m}|A(0)| p_{m+1}, \ldots, p_{n}\right\rangle_{\alpha_{m+1} \ldots \alpha_{n}}^{i n, \text { con }} \\
= & F_{\underline{\alpha}}^{A}\left(s_{i j}+i \varepsilon, t_{r s}-i \varepsilon, s_{k l}+i \varepsilon\right), \quad i<j \leq m<k<l \leq n \\
t_{r s}= & \left(p_{r}-p_{s}\right)^{2}, \quad r \leq m<s \leq n
\end{aligned}
$$

There are Watson relations between the S-matrix and the formfactors. In the $\mathrm{d}=1+3$ dispersion theory setting it is well known that the cuts below the inelastic threshold of $\left\langle 0|A(0)| p_{1}, p_{2}\right\rangle$ is related to the partial wave phase shifts in that elastic region. In a factorizing $d=1+1$ theory these Watson relations can be written down in general:

$$
\begin{aligned}
& F_{\alpha_{1} \ldots \alpha_{n}}^{A}\left(s_{i j}+i \varepsilon\right)=\left\langle 0|A(0)| p_{1}, \ldots . p_{n}\right\rangle_{\alpha_{1} \ldots \alpha_{n}}^{i n} \\
&=\sum_{\text {out }}\langle 0|A(0)| \text { out }\rangle\left\langle\text { out } \mid p_{1}, \ldots, p_{n}\right\rangle_{\alpha_{1} \ldots \alpha_{n}}^{\text {in }} \\
& \curvearrowright F_{\alpha_{1} \ldots \alpha_{n}}^{A}\left(s_{i j}+i \varepsilon\right)=F_{\alpha_{1}^{\prime} \ldots \alpha_{n}^{\prime}}^{A}\left(s_{i j}-i \varepsilon\right) S_{\alpha_{1} \ldots \alpha_{n}}^{\alpha_{1}^{\prime} \ldots \alpha_{n}^{\prime}}\left(s_{i j}\right)
\end{aligned}
$$

and for the mixed formfactors32:

$$
\begin{aligned}
& F_{\underline{\alpha}}^{A}\left(s_{i j}+i \varepsilon, t_{r s}-i \varepsilon, s_{k l}+i \varepsilon\right) \\
= & S_{\alpha_{1}^{\prime} \ldots \alpha_{m}^{\prime}}^{\alpha_{m} \ldots \alpha_{1}}\left(s_{i j}\right) F_{\underline{\alpha}^{\prime}}^{A}\left(s_{i j}-i \varepsilon, t_{r s}+i \varepsilon, s_{k l}-i \varepsilon\right) S_{\alpha_{m+1} \ldots \alpha_{n}}^{\alpha_{n}^{\prime} \ldots . \alpha_{m+1}^{\prime}}\left(s_{k l}\right)
\end{aligned}
$$

Using the uniformazing $\theta^{\prime} s$ this is like a generalized quasiperiodicity property on $\theta$-strips for the F's (instead of the periodicity of $\mathrm{S}$ ). The first who considered formfactors beyond two-particles [18] and presented a system of 
axioms for their calculation was Smirnov [15] Following a recent presentation by Babujian, Fring and Karowski [16] in a more standard field theoretic setting (LSZ+ dispersion theory), the formfactor program for the construction of $\mathrm{d}=1+1 \mathrm{QFT}$ is as follows. Introduce the orderd formfactors:

$$
f_{\underline{\alpha}}^{A}\left(\theta_{1}, \ldots, \theta_{n}\right):=\left\langle 0|A(0)| p_{1}, \ldots, p_{n}\right\rangle_{\underline{\alpha}}^{i n}, \quad \theta_{1}>\ldots>\theta_{n}
$$

and define the value for reordered $\theta^{\prime} s$ by analytic continuation (starting with this ordering in the physical region). Demand that the f's fulfill the following properties:

- (i) $f_{\ldots i j \ldots}^{A}\left(\ldots, \theta_{i}, \theta_{j}, \ldots\right)=f_{\ldots j i \ldots}^{A}\left(\ldots, \theta_{j}, \theta_{i}, \ldots\right) S_{i j}\left(\theta_{i}-\theta_{j}\right) \quad \forall \theta^{\prime} s$

- (ii) $f_{12 \ldots n}^{A}\left(\theta_{1}+i \pi, \theta_{2}, \ldots, \theta_{n}\right)=f_{2 \ldots n 1}^{A}\left(\theta_{2}, \ldots, \theta_{n}, \theta_{1}-i \pi\right)$

- (iii) $f_{1 \ldots n}^{A}\left(\theta_{1}, \ldots \theta_{n}\right) \underset{\theta_{1} \rightarrow \theta_{2}+i \pi}{\approx} \frac{2 i}{\theta_{1}-\theta_{2}-i \pi} C_{12} f_{3 \ldots n}^{A}\left(\theta_{3}, \ldots, \theta_{n}\right)\left(1-S_{2 n} \ldots S_{23}\right)$

where $C_{\alpha \beta}=\delta_{\bar{\alpha} \beta}$ is the charge conjugation matrix.

Here we have not mentioned the poles from bound states (states which appear by the previous fusion) since they are automatically entering the formfactors via the S-matrix. The word "axiom" in the context of this paper has the significance of working hypothesis i.e. an assumption which receives its legitimation through its constructive success. Physical principles on the other hand, as the spectral and causality properties of general QFT, will not be called axioms. Our main aim is to show how one can reduce the above axioms of the bootstrap-formfactor approach to the principles of QFT and thereby recuperate the unity of this nonperturbative approach with the rest of QFT.

The conceptually somewhat unusual property is the "symmetry" property (i). Here one should bear in mind that from the point of view of the LSZ formulation $\mathrm{f}$ is an auxiliary object to which the statistics property under particle exchange does not apply (it would apply to the original matrixelement). The above exchange property for $f$ is a statement about analytic continuation. The statistics of incoming particle is only used in order to get the charges (i.e. the tensor factors) into the same $j-i$ order as the analytically interchanged $\theta^{\prime} s$. Following BFK [16] let us first remind ourselves of the standard argument for (i) in somewhat detail. For the special case $\left\langle 0|A(0)| \theta_{1} \theta_{2}\right\rangle^{e x} e x=i n$, out it is evident that:

$$
\lim _{\varepsilon \rightarrow 0} F\left(s_{12} \pm i \varepsilon\right)=\left\{\begin{array}{l}
\left\langle 0|A(0)| \theta_{1} \theta_{2}\right\rangle^{\text {in }} \\
\left\langle 0|A(0)| \theta_{1} \theta_{2}\right\rangle^{\text {out }}
\end{array}\right.
$$


i.e. there is one analytic masterfunction $f(z)$ (assuming identical particles) with different boundary values on the $s \geq 4 m^{2}$ cut having the in, out interpretation. Assuming Bose statistics, the physical matrix elements on the right hand side are symmetric under the interchange of the $\theta^{\prime} s$. In terms of the uniformization variable $\theta_{12}$ in $F$ the transition from in $\rightarrow$ out means a change of sign via analytic continuation i.e. without changing the charge quantum numbers $\alpha$ i.e. the position of the tensor factors. After accomplishing this last step by the bose commutation relation the negative $\theta_{12}$ formfactor $F\left(\theta_{21}\right)$ can according to the definition 38 be identified with $f_{21}\left(\theta_{2}, \theta_{1}\right)$ and the relation

$$
\left\langle 0|A(0)| \theta_{2} \theta_{1}\right\rangle^{\text {out }} S\left(\left|\theta_{1}-\theta_{2}\right|\right)=\left\langle 0|A(0)| \theta_{1} \theta_{2}\right\rangle^{\text {in }}
$$

agrees with 38 The generalization to ${ }^{\text {out }}\left\langle\theta_{3} \ldots \theta_{n}|A(0)| \theta_{1} \theta_{2}\right\rangle_{\text {con }}^{e x}$ has a problem because replacing in by out means passing from time-ordering to anti-timeordering but the LSZ scattering theory produces boundary terms contributing to the connected part. Although they are absent for theories in which the number of in-particles are conserved, it is unclear what property of general QFT is bringing about (i) through specialization to factorizing $\mathrm{d}=1+1$ models.

On the other hand (ii) and (iii) are consequences of the following standard crossing formula [16] which relate the connected part of the generalized formfactors to the analytic master function $f$ :

$$
\begin{aligned}
& \overline{1}\left\langle 0|A(0)| p_{2}, \ldots, p_{n}\right\rangle_{2 \ldots n}^{i n} \\
= & \sum_{j=2}^{n}{ }_{\overline{1}}^{n}\left\langle p_{1} \mid p_{j}\right\rangle_{j} f_{2 . . \hat{j} . . n}^{A} S_{2 j} \ldots S_{j-1 j}+f_{12 \ldots n}^{A}\left(\theta_{1}+i \pi_{-} \ldots, \theta_{n}\right) \\
= & \sum_{j=2}^{n}{ }_{\overline{1}}^{n}\left\langle p_{1} \mid p_{j}\right\rangle_{j} f_{2 . . \hat{j} . . n}^{A} S_{j n} \ldots S_{j j+1}+f_{2 \ldots n 1}^{A}\left(\ldots, \theta_{n} \theta_{1}-i \pi_{-}\right)
\end{aligned}
$$

The fastes way to understand this is to draw the corresponding graphs and remember that a positive energy particle crosses into a negative energy antiparticle. Successive application leads to a formula which expresses the formfactors in terms of the analytic auxiliary function $f$.The analytic part of this relation gives (ii) whereas the $\delta$-function part is responsible for (iii). A proof that these properties do not only insure TCP-invariance (weak locality) but also Einstein causality can be given by using JLD spectral representations [19] However the direct derivation of the bootstrap-formfactor 
axioms from the principles of QFT was hitherto not achieved. It is part of the complicated and incomplete momentum space analyticity problem. Even the derivation of forward dispersion relations in particle physics took several years, not to mention the derivation of the analytic aspects 9 of crossing symmetry which remained utterly incomplete. It is precisely at this point where our modular localization approach shows its strength. To anticipate one result, it shows that the crossing symmetry is a kind of strengthened TCPproperty and that the cyclicity it leads to is identical to the KMS-temperature (三Hawking -Unruh temperature in this special case) characterization of the (Rindler-)wedge based Hawking-Unruh effect. From our point of view the most valuable result is that it opens for the first time the way to a new constructive iterative (but not perturbative) approach to non-quantization non-Lagrangean based QFT. My confidence that this may amount to more than just another fashion rests on the observation that the tool of modular localization comes from a refinement of TCP which, as anybody will immediately admit, the central structure of local QFT.

\subsection{Modular Theory and the Formfactor Program}

In this section we will analyze the formfactor program from the viewpoint of modular localization. To avoid complications we start with theories which have a diagonal S-matrix. A prototype is the Ising field theory with $S^{(2)}=$ $-1, S=K$ where $\mathrm{K}$ is the $Z_{2}$ Klein twist of the previous section. We look first for a weakly local (not necessarily local) field in the TCP class associated to this simple S-matrix. For this purpose we modify the Fermi-creation and annihilation operators $a_{i n}^{\#}$ of the free Majorana field associated to the Ising field theory in order to obtain the bosonic $\mathbf{Z}_{2}$ commutation relations. With some experience [20] one immediately writes the following expressions:

$$
\begin{gathered}
b(\theta)=: a_{i n}(\theta) e^{-i \pi \int_{-\infty}^{\theta} a_{i n}^{*}(\theta) a_{i n}(\theta) d \theta}: \\
c^{*}(\theta)=: a_{i n}^{*}(\theta) e^{i \pi \int_{\theta}^{\infty} a_{i n}^{*}(\theta) a_{i n}(\theta) d \theta}: \\
B(x) \quad: \quad \frac{1}{\sqrt{2 \pi}} \int\left\{e^{-i p x} b(\theta)+e^{i p x} c^{*}(\theta)\right\} d \theta \\
\curvearrowright \quad \Theta B(x) \Theta=B^{*}(-x), \quad \Theta=S_{\text {Ising }} \cdot \Theta_{0}, \quad \Theta_{0} \equiv \Theta_{\text {in }}
\end{gathered}
$$

\footnotetext{
${ }^{5}$ Only together with the (mass shell) analytic properties the crossing symmetry aquires a physical content.
} 
This field creates wedge localized vectors in the n-particle projections of $\mathcal{D}(W)=H_{R}+i H_{R}$ :

$$
B^{\#}\left(x_{1}\right) \ldots B^{\#}\left(x_{n}\right) \Omega, \quad x_{i} \in W
$$

These $b^{\#} s$ produce the $S_{\text {Ising }}$ S-matrix:

$$
\begin{aligned}
b^{*}\left(\theta_{1}\right) \ldots . b^{*}\left(\theta_{n}\right) \Omega & =S_{\text {Ising }} a^{*}\left(\theta_{1}\right) \ldots . a^{*}\left(\theta_{n}\right) \Omega \\
b^{\#}(\theta) & =S_{\text {Ising }} a^{\#}(\theta) S_{\text {Ising }}^{*}
\end{aligned}
$$

and fulfill the Zamolodchikov algebra:

$$
\begin{aligned}
b(\theta) b^{*}\left(\theta^{\prime}\right) & =S^{(2)-1}\left(\theta-\theta^{\prime}\right) b^{*}\left(\theta^{\prime}\right) b(\theta)+\delta\left(\theta-\theta^{\prime}\right) \\
b(\theta) b\left(\theta^{\prime}\right) & =S^{(2)}\left(\theta-\theta^{\prime}\right) b\left(\theta^{\prime}\right) b(\theta)
\end{aligned}
$$

Operators of this kind have been used a long time ago in order to exemplify the fact that massive $\mathrm{d}=1+1$ theories describe "statistical schizons" 20 in distinction to conformal field theory, where the statistics (in form of field commutation relations) are inexorably linked with the fusion law of charges ${ }^{6}$. Recently Fring [21] and Lashkevich 222 constructed these operators using the realization of anyionic deformations [20] of Fock space $d=1+1$ creation- and annihilation operators $a^{\#}(p)$ as a guiding principle. It was through discussions I had with Lashkevich that I later recognized that there may be an interesting relation between the above Zamolodchikov algebra and certain auxiliary operators in my modular localization approach.

The above expressions may be generalized to factorizable S-matrices which are diagonal.. The corresponding b-operators are then of the form:

$$
b(\theta)=a_{i n}(\theta) e^{i \int_{-\infty}^{\theta} \delta_{s c}\left(\theta-\theta^{\prime}\right) a_{i n}^{*}\left(\theta^{\prime}\right) a_{i n}\left(\theta^{\prime}\right) d \theta^{\prime}}
$$

where $\delta_{s c}(\theta)$ is the scattering phase shift. Again the TCP invariant operators and the wedge localization through the $\check{S}$-domain is analogous to the

\footnotetext{
${ }^{6}$ For this reason the widespread use of the terminology "bosonization" in conformal QFT is unfortunate, but appropriate in $\mathrm{d}=1+1$ massive theories. Whereas "bosonization" (or "fermionization") in conformal QFT means that the current algebra admits fermionic superselection sectors, in the massive case the same sector allows different "pseudo"statistical descriptions since schizons only have intrinsic charges but not intrinsic statistics. A periodic table of elements in a $d=1+1$ world does not require fermions.
} 
constant case. From those wedge localized n-particle states one reads off the formfactors of the would be local field. The solution is unique.

The main observations which links the bootstrap-formfactor axiomatics (i) (ii) and (iii) with modular localization are contained in the following statements:

- The TCP-covariant (not necessarily local, but weakly local) fields $B(x)$ with $x=r(\sinh \chi, \cosh \chi) \in W$ which generate a right wedge algebra $\mathcal{A}(W)$ equipped with the global vacuum state (i.e. that state which is the vacuum with respect to the global algebra $\mathcal{A}$ ) result in a KMStemperature state with the Hawking-Unruh temperature $\beta=2 \pi$. The KMS boundary condition for a correlation function of $B^{\prime} s$ together with local fields $A(x), x \in W$ :

$$
\begin{aligned}
& \left\langle 0\left|B\left(r_{1}, \chi_{1}\right) B\left(r_{2}, \chi_{2}\right) \ldots A(x) \ldots B\left(r_{n}, \chi_{n}\right)\right| 0\right\rangle \\
= & \left\langle 0\left|B\left(r_{2}, \chi_{2}\right) . . A(x) \ldots B\left(r_{n}, \chi_{n}\right) B\left(r_{1}, \chi_{1}+2 \pi i\right)\right| 0\right\rangle
\end{aligned}
$$

is equivalent to the cyclicity property (ii) (the $f^{A}$ is obtained from the connected part of the B-correlation function). The $2 \pi$ strip-analyticity in the $\chi^{\prime} s$, which is provided by the KMS-theory, translates into momentum space analyticity for the rapidity variables $\theta_{i}$ of $f^{A}$. In particular the analyticity and the crossing symmetry of the S-matrix is a consequence of this temperature structure 47 for $A=1$ e.g.:

$$
\begin{aligned}
& \left\langle 0\left|B\left(r_{1}, \chi_{1}+i \pi\right) B\left(r_{2}, \chi_{2}\right) B^{*}\left(r_{3}, \chi_{3}\right) B^{*}\left(r_{4}, \chi_{4}\right)\right| 0\right\rangle \\
= & \left\langle 0\left|B\left(r_{2}, \chi_{2}\right) B^{*}\left(r_{3}, \chi_{3}\right) B^{*}\left(r_{4}, \chi_{4}\right) B\left(r_{1}, \chi_{1}-i \pi\right)\right| 0\right\rangle \\
\Leftrightarrow & S(\theta)=S(i \pi-\theta)
\end{aligned}
$$

- The (improper) state vectors $B\left(r_{1}, \chi_{1}\right) \ldots B\left(r_{n}, \chi_{n}\right) \Omega$ are boundary values of "half-strip" analytic vectors:

$$
B\left(r_{1}, z_{1}\right) \ldots . B\left(r_{n}, z_{n}\right) \Omega, \quad \pi>\operatorname{Im} z_{n}>\ldots . \operatorname{Im} z_{1}>0
$$

Upon taking the n-particle component this property translates into the half strip analyticity of the vectors:

$$
b^{*}\left(z_{1}\right) \ldots . b^{*}\left(z_{n}\right) \Omega, \quad z_{i}=\theta_{i}+i \vartheta_{i}
$$

As a result of the Zamolodchikov commutation relations of the $b^{\# \prime} s$, we find that there is one half strip-analytic "master" state vector $\psi\left(z_{1}, \ldots . z_{n}\right)$, 
whose different boundary values correspond to different operator orderings:

$$
\lim _{\pi>\operatorname{Im} z_{P(1)}>\ldots>\operatorname{Im} z_{P(n)}>0} \psi\left(z_{1} \ldots z_{n}\right)=b^{*}\left(\theta_{P(1)}\right) \ldots b^{*}\left(\theta_{P(n)}\right) \Omega
$$

The analytic structure in rapidity space has an intriguing similarity with the analytic x-space structure known from Wightman's formulation of QFT. The reason is of course the close connection between the L-boost variable $\chi$ and the rapidity $\theta$.

- The above state vectors generate the n-particle component of the modular localization subspace:

$$
\begin{gathered}
P_{n} \check{S} B\left(x_{1}\right) \ldots B\left(x_{n}\right) \Omega=P_{n} B^{*}\left(x_{n}\right) \ldots B^{*}\left(x_{1}\right) \Omega \\
\Leftrightarrow \check{S} c^{*}\left(\theta_{1}\right) \ldots c^{*}\left(\theta_{n}\right) \Omega=b^{*}\left(\theta_{n}\right) \ldots b^{*}\left(\theta_{1}\right) \Omega \\
\curvearrowright c^{*}\left(\theta_{1}\right) \ldots c^{*}\left(\theta_{n}\right) \Omega+b^{*}\left(\theta_{n}\right) \ldots b^{*}\left(\theta_{1}\right) \Omega \in H_{R}^{(n)}(W)
\end{gathered}
$$

Here we used the relation 45 i.e. the factorizability of the theory. As in the free case, the closed real subspace $H_{R}(W)$ represents the encoding of the complex dense modular localization space associated with $W$. Note that this last discussion used the factorization structure.

The generalization to charged particles and to halfinteger Lorentz spin is straightforward but the case of nondiagonal S-matrices gives rise to additional problems. The modular localization equation for the real subspace $H_{R}^{(n)}(W)$ is now:

$$
\begin{aligned}
& \check{S} \int \psi_{\alpha_{1} \ldots \alpha_{n}}\left(\theta_{1}, \ldots \theta_{n}\right)\left|\theta_{1}, \ldots \theta_{n}\right\rangle_{i n}^{\alpha_{1} \ldots \alpha_{n}} \\
= & \int\left(\bar{\psi}_{\alpha_{1}^{\prime} \ldots \alpha_{n}^{\prime}} S_{\alpha_{1} \ldots \alpha_{n}}^{\alpha_{1}^{\prime} \ldots \alpha_{n}^{\prime}}\right)\left(\theta_{1}+i \pi \ldots \theta_{n}+i \pi\right)\left|\theta_{1}, \ldots \theta_{n}\right\rangle_{i n}^{a_{1} \ldots \alpha_{n}} \\
= & \int \psi_{\alpha_{1} \ldots \alpha_{n}}\left(\theta_{1}, \ldots \theta_{n}\right)\left|\theta_{1}, \ldots \theta_{n}\right\rangle_{i n}^{\alpha_{1} \ldots \alpha_{n}} \\
\curvearrowright & \left(\bar{\psi}_{\alpha_{1}^{\prime} \ldots \alpha_{n}^{\prime}} S_{\alpha_{1} \ldots \alpha_{n}}^{\alpha_{1}^{\prime} \ldots \alpha_{n}^{\prime}}\right)\left(\theta_{1}+i \pi \ldots \theta_{n}+i \pi\right)=\psi_{\alpha_{1} \ldots \alpha_{n}}\left(\theta_{1}, \ldots \theta_{n}\right)
\end{aligned}
$$

This "S-reality" equation seems to be a new mathematical structure as far as the mathematical physics literature is concerned. In the formulation of 
crossing symmetry the charge multiplicity indices $\alpha$ must be replaced by their conjugate values:

$$
S_{\alpha_{1} \alpha_{2}}^{\beta_{1} \beta_{2}}\left(\theta_{1}-\theta_{2}\right)=S_{\alpha_{2} \beta_{2}}^{\bar{\alpha}_{1} \beta_{2}}\left(\theta_{2}-\left(\theta_{1}-i \pi\right)\right)
$$

In the case of nondiagonal $S$ one does not have operators $B(x)$ at ones disposal. It turns out that analogous to the Bethe Ansatz inspired solution of this problem [16]in the formfactor approach, the modular wedge localization (i.e. the S-reality) entails a natural Bethe Ansatz structure which permits an explicit description of the space $H_{R}^{(n)}(W)$. The latter is indispensable for a realization of the Zamolodchikov algebra in the incoming Fock space and the construction of local fields within the formfactor program. These matters will be taken up in a separate paper [19]. There we will also present the new rich structures which result from intersections of wedge spaces in order to describe the localization spaces e.g. of the compact double cone regions. Although the modular structure of wedge localized von Neumann algebras in QFT as we used it here may be found in the literature, we do not expect most of our readers to know it. Therefore we decided to present the modular material in a broader context ??.

The coordinate-free point of view of the net theory suggests that the formfactor program may not be the most efficient and natural way to relate an S-matrix with local fields i.e. the inverse problem of the net field theory: given the modular invariant $S$ of the net, reconstruct the net. Since an S-matrix is not associated to a particular field but rather is an invariant of a local equivalence class or a net, a direct construction (in the spirit of the functorial construction for free systems in the introduction) of the net instead of individual fields may be simpler than the rather cumbersome formfactor program. Such an algebraic approach would then consist of two parts, the conceptual problems related to the modular wedge localization of state vectors in Fock space i.e. TCP, antiparticles, crossing symmetry etc. and the ascend to local nets of subspaces and associated nets of von Neumann algebras.

Apart from the new analytic modular structure, the wedge localization equation is reminiscent of Yang's use of the Bethe Ansatz idea [17]. Our modular localization method therefore suggests that a suitably generalized Bethe idea may be a valuable tool in a new constructive approach to algebraic QFT (and not just for factorizable models). Related to this is the hope that our approach may lead to an explicit Fock space representation of the 
Zamolodchikov algebra (beyond the above diagonal cases) also in nondiagonal factorizable models, and that one meets analogues of this algebraic structure (which is somewhere intermediate between the algebras generated by the Heisenberg fields and that of the incoming fields and hence may be viewed as an algebraic QFT counterpart of the fictitious [3] interaction picture) in general local quantum physics.

\section{Open Ends and Outlook}

The really difficult problem in a constructive approach build on modular localization is the passing from the net of localized subspaces to a net of von Neumann subalgebras (educated guess: subfactors of von Neumann type $I I I_{1}$ as in the free field case). Here the most important issue is uniqueness. The net of localized subspaces is uniquely determined by the TCP-operator. Therefore the question of uniqueness of nets of operator algebras can be rephrased as: does the weak locality equivalence class contain maximally only one local Borchers class? We think that by a judicial use of spacelike (anti)commutativity, a given S-matrix $(\Leftrightarrow$ a given $\Theta$ ) will maximally allow (up to isomorphisms) one field system or algebraic net. The only argument we have in the moment is : $S=1 \curvearrowright$ free field Borchers class [19]. The essential step in the argument is the use of crossing symmetry and the Watson relations which link physical cuts in the analytically continued formfactors (matrix elements of a representative local field in the basis of the incoming multiparticle spaces) to the S-matrix. The fact that analytic on shell p-space properties, as one needs them for such arguments (e.g. crossing symmetry as an analyticity statement and not just a suggestive formal statement abstracted from LSZ extrapolation formulas), are difficult to obtain from the locality and spectral principles of QFT one can try to counteract by (as in the $\mathrm{d}=1+1$ factorizable case) assuming "maximal analyticity" i. e. only taking physically motivated singularities into account. On the one hand this is certainly reasonable in a constructive approach and on the other hand we expect that the same modular theory which underlies the modular localization approach will also lead to a much better understanding of on shell analytic p-space properties. The present analytic techniques result from the so-called JLD-representation for matrix elements of causal commutators. We expect the exploration of modular localization concepts to give more powerful analytic results. 
Some speculative remarks on the problem of associating a net of von Neumann algebras with a net of localization spaces may be helpful at this point. In the theory of operator algebras the Araki-Connes concept of the "natural cone" allows to construct a von Neumann algebra from the knowledge of the split of $H_{R}$ into positive cones $\pm C_{+}$:

$$
H_{R}=C_{+}-C_{+}
$$

In the case of factorizable models the modular localization principle which leads to $H_{R}(W)$ - and $H_{R}$ (double cone)-subspaces uses the real (on shell) particle conservation and gave rise to (albeit new and subtle) quantum mechanical on shell Bethe Ansatz problems. However the local fields (or nets of von Neumann algebras) in such theories are known to have a very rich (nonquantum mechanical!) virtual ("off-shell") particle creation and annihilation structure. The same is expected in a theory of "free" anyons and plektons as opposed to free Bosons and Fermions. According to the arguments presented in this paper, this richness must occur in this last (unfortunately poorly understood) step from modular localized subspaces to (Einstein-) local algebras $^{7}$. Connected with this is the already mentioned uniqueness problem i.e. the inverse scattering problem for local nets, which we will investigate in a future publication [19].

Finally we want to make some speculative remarks on how one imagines an iterative approach which unlike perturbation theory (which uses interaction densities expressed in the form of Wick-polynomials and their timeordering) is based on modular concepts. One would start with a Heisenberg Ansatz for a relativistic S-matrix:

$$
S^{(0)}=e^{i \eta}, \quad \eta=\sum \int \eta_{n}\left(x_{1} \ldots x_{2}\right): \phi_{i n}\left(x_{1}\right) \ldots \phi_{i n}\left(x_{n}\right):
$$

where the coefficient function should be Poincaré-invariant and cluster-connected, but yet without those complicated analytic p-space properties of multidimensional dispersion theory. In order to make at least some formal intuitive contact with the standard perturbative implementation of interaction via invariant free field polynomials one may choose for $\eta$ the same expression. This Ansatz already assures (via $\Theta$ and $J$ ) the modular wedge localization ( at almost no analytic costs) which leads to mass shell analyticity in certain rapidity variables (which form families corresponding to the family of wedges)

${ }^{7}$ This is also step from which I would expect a profound understanding of on shell crossing symmetry. 
as in the factorizable case ??. However the nontriviality of intersections of wedge spaces and the required existence of a map from the net of modular localized subspaces to a net of von Neumann algebras suggests to iteratively correct $S^{(0)}$ by $S^{(1)}, S^{(2)}$..etc. in order to achieve an increasing amount of localization 8 . Unlike the bootstrap-formfactor problem of the previous section for which a candidate for a "local", crossing symmetric S-matrix fulfilling the "maximal" p-space analyticity was known at the start of the formfactor program, such a contemplated iterative approach based on modular localization would constitute a true ("heterotic field theory-S-matrix") mixed bootstrap approach. It would be at least as removed from perturbation theory and functional integrals as the bootstrap-formfactor program is from Lagrangian QFT. The interaction could then receive its name not from Lagrangians but rather from the e.g. polynomial Ansatz for $\eta$.

At this point the question arises what, if any, is the relation between this modular approach and the standard one build on quantization. Here it is helpful to think of a kind of field theory-adapted "Murphy's law": if there is neither a proof nor a an intrinsic structural reason for a conjecture in QFT, then it is wrong. Although this law does not follow from the physical principles, I do not know a single exception to it in QFT. So the idea, that behind the renormalized Gell-Mann Low formula or functional integrals (even with all their instanton corrections!) or the Bogoliubov-Shirkov axiomatics in terms of a space-time localized formal expression $S(g)$, there is an interacting theory based on those concepts would be an illusion, except for those low-dimensional models ( $\varphi_{2}^{4}$ etc. where there is a connection through Borel resummability) for which one has a proof. Perturbation theory remains an infinitesimal deformation theory with no possibility for globalization. Despite its non-existence (invoking Murphy's law) of $S(g)$, from a formal physical point of view it is close to the modular invariant $S$ (section 4 of [1]. Whereas standard perturbation theory maintains the linear structures as locality in every order but links unitarity with the non-perturbative existence (futile according to Murphy's law in QFT), any inductive approach based on modular properties is unitary in each step of the induction, but acquires sharp locality only in the limit (which is also the limit in which. the modular $S$ acquires the scattering interpretation).

\footnotetext{
${ }^{8}$ The problem seems to be vagely reminiscent of a selfconsistent Hartree-Fock iteration with the iteratively improved interaction being the analog of the $S^{(n) \prime} s$, and the zero order bilinear (mean field) interaction corresponding to an $S^{(0)}$ in the form of a Heisenberg ansatz57.
} 
The present modular framework is not applicable to zero mass theories for which the LSZ incoming fields vanish i.e. a scattering matrix cannot be defined ("Infraparticles" as opposed to Wigner particles). Whereas for e.g. chiral conformal theories this is no problem since they are scale-invariant limits of massive theories, theories as QED (which presumably do not allow such a limiting description) require conceptual modifications. The modular approach is uncompromising but thankful for new conceptional challenges. It flourishes on the weaknesses, paradoxes and contradictions of the standard approach. Even at the risk of sounding immodest, one may hope to overcome the narrowing of QFT at the beginning of the $70^{i e s}$, when (as a result of enforcing classically based concepts as the "gauge principle" via quantization) very unfortunately noncommutative real time field theory was forced to become a footnote of euclidean field theory. Besides recent (in my opinion artificial) attempts to get out of the euclidean malaise by compensating the loss with a doses of noncommutative geometry? there has been a renewed interest in real time QFT. In addition to the formfactor-bootstrap program presented in this work, this is illustrated by recent progress on QFT in curved space-time for which the euclidean approach is physically senseless 23] (albeit mathematically interesting). We hope that our ideas on modular localization may also contribute to find a way out of the present stalemate and particularization of QFT. In any case our confidence at the moment is more based on its unifying point of view and its fundamental modular structure leading to new mathematical equations with the promise of a deep relation to Bethe Ansatz structures. Anybody with some knowledge of nonperturbative QFT will have no problem in recognizing that one is dealing here with the most fundamental structures which QFT has to offer.

Acknowledgments: I am indebted to Andreas Fring and Michael Karowski for their patience in explaining some intricate points in the formfactor program and for letting me know their explicite multi-formfactor constructions for certain models with non-diagonal S-matrix prior to publication. I also thank Hans-Werner Wiesbrock for his active interest in these constructive applications of modular ideas as well as Karl-Henning Rehren for a critical reading of a preliminary version of the manuscript.

\footnotetext{
${ }^{9}$ Instead of trying to escape the euclidean trap by physical brute force methods (employing ideas from nomcommutative geometry) one should remember that QFT has a natural noncommutative structure and allows for a euclidean formulation and a Feynman-Kac representation only under very special circumstances.
} 


\section{References}

[1] B. Schroer, "Wigner Representation Theory, Localization and Statistics", hep-th 968092

[2] E.P.Wigner, Ann. Math.40, 149 (1939)

[3] R.Haag, "Local Quantum Physics" Springer Verlag 1992

[4] B. Schroer, "Motivations and Physical Aims of Algebraic QFT", hep-th 968083, to appear in the March 1997 issue of Ann. of Physics.

[5] R. Brunetti, D. Guido and R. Longo, to appear

[6] J.J. Bisognano and E.H. Wichmann, J. Math. Phys. 16, 985 (1975); 17, 303 (1976)

[7] G.L. Sewell, Phys. Rev. Lett. 79A, 23 (1980)

[8] R.F. Streater and A.s. Wightman, "PCT, Spin and Statistics and All That" New York, Benjamin 1964

[9] A.B. Zamolodchikov, Int. J. Mod. Phys. A1 (1989) 4235

[10] B. Schroer, T.T. Truong and P.Weisz, Phys. Lett. 63B (1976) 422

[11] B. Berg, M. Karowski, H.J. Thun, T.T. Truong and P. Weisz, Phys. Lett. 67B (1977) 321

[12] A.B. Zamolodchikov, Comm. Math. Phys.55 (1977) 183, ITSP (1977) 12.

[13] V. Kurak and J.A. Swieca,Phys. Lett.92 (1979) 289

[14] V. Jones, Vaughn F.R. Jones, American Math Society publication No 80, 1991

[15] F.A. Smirnov, Adv. Series in Math.Phys. 14, World Scientific1992

[16] H. Babujian, A. Fring and M. Karowski, "Form Factors of the $S U(N)-$ Chiral Gross Neveu Model", in preparation.

[17] C.N. Yang, Phys.Rev.Lett. B86, (1977) 209 
[18] M. Karowski and P. Weisz, Nucl.Phys. B139 (1978) 445

[19] B. Schroer and H.W. Wiesbrock, in preparation

[20] B. Schroer and J.A. Swieca, Nucl. Phys. B121 (1977) 505

[21] A. Fring, I.J.of Mod. Phys. A 11, (1996) 1337

[22] M. Yu Lashkevich, "Sectors of Mutually Local Fields in Integrable Models of QFT" Landau Institute Preprint, hep-th 9406118

[23] R. Brunetti and K. Fredenhagen, "Interacting Quantum Field Theories in Curved Space: Renormalizability of $\varphi^{4 "}$ 